\title{
APLICAÇÃO DO MÉTODO DE LAWSHE PARA VALIDAÇÃO DE ITENS DE UM QUESTIONÁRIO SOBRE A SATISFAÇÃO DE CLIENTES DE UM SUPERMERCADO EM CAMPOS DOS GOYTACAZES, RJ
}

\author{
Juliany Carvalho de Souza ${ }^{1}$, Maria Clara Campos Alves ${ }^{1}$, Francisco de Assis Léo \\ Machado $^{1,2 *}$, Aldo Shimoya ${ }^{1}$ \& Paulo Sérgio Peçanha Luna ${ }^{3}$
}

\section{RESUMO}

SOUZA, J. C.; ALVES, M. C. C.; MACHADO, F. A. L.; SHIMOYA, A.; LUNA, P. S. P. Aplicação do método de Lawshe para validação de itens de um questionário sobre a satisfação de clientes de um supermercado em Campos dos Goytacazes, RJ. Perspectivas Online: Exatas \& Engenharia, v.10, n.27, p.42- 53, 2020.

A satisfação dos clientes é um dos fatores principais para o sucesso de uma empresa no mercado competitivo. O objetivo do trabalho foi validar os itens do questionário sobre a satisfação, segundo a percepção dos clientes, dos produtos e serviços oferecidos por um supermercado, no município de Campos dos Goytacazes RJ. A elaboração do questionário foi realizada com base em consultas de literatura científica como artigos de periódicos, teses, dissertações e congressos. Para definir os itens do questionário foram selecionados 20 trabalhos, de onde foram obtidos 30 itens distribuídos em cinco dimensões: "Estrutura" (8 itens), "Atendimento" (9 itens), "Produtos" (7 itens), "Adicionais" (4 itens) e "Segurança" (2 itens). Foi utilizado o método de Lawshe para validação de cada item do questionário, onde cada item oferecia as seguintes opções de respostas: (1) não essencial, (2) essencial, (NS) não sei. No método de Lawshe foi calculado o CVR para cada item, utilizando o nível de $5 \%$ de probabilidade. $\mathrm{O}$ critério do item a ser mantido no questionário foi CVRcalculado > CVRcrítico, caso o CVRcalculado < CVRcrítico o item é excluído. O questionário foi aplicado a 48 clientes. Dos trinta itens avaliados, somente os itens "Serviço de entrega em domicílio" e "Ações em datas comemorativas (sorteios e brindes)", da dimensão "Adicionais", foram excluídos. Nas outras dimensões, os consumidores valorizaram mais os itens "Limpeza e higiene do ambiente", "Agilidade no atendimento no caixa", "Funcionários dispostos a informar e ajudar", "Preços justos" e "Produtos de qualidade", indicando que os itens mantidos no questionário podem ser utilizados para auxiliar futuras pesquisas de avaliação da satisfação de clientes de supermercados.

Palavras-chave: Supermercado; Questionário; Satisfação; Lawshe.

Persp. Online: exatas \& eng., Campos dos Goytacazes, 27 (10) 43 - 53 - 2020 seer.perspectivasonline.com.br 


\begin{abstract}
Customer satisfaction is one of the main factors for the success of a company in the competitive market. This work aimed at validating the items of a questionnaire on satisfaction, regarding the perception of customers, with the products and services offered by a supermarket, in the municipality of Campos dos Goytacazes, Rio de Janeiro State, Brazil. The questionnaire was prepared based on searches in the scientific literature, such as journal articles, theses, dissertations, and conferences. Twenty studies were chosen to define the items of the questionnaire, from which 30 items distributed in five dimensions were obtained: "Structure" (8 items), "Service" (9 items), "Products" (7 items), "Additional" (4 items), and "Safety" (2 items). The Lawshe method was adopted to validate each item of the questionnaire, in which each one presented the following response options: (1) not

essential, (2) essential, (DNK) do not know. In the Lawshe method, the CVR for each item was calculated using the 5\% probability level. The criterion for the item to be maintained in the questionnaire was CVRcalculated > CVRcritical; in case CVRcalculated $<$ CVRcritical, the item is excluded. The questionnaire was applied to 48 customers. Among the 30 items evaluated, only the items "Home delivery service" and "Actions on commemorative dates (draws and gifts)", of the "Additional" dimension, were excluded. In the other dimensions, consumers appreciated more the items "Cleaning and hygiene of the place", "Agility at the cashier service", "Employees prepared to inform and help", "Fair prices", and "Quality products", stating the items maintained in the questionnaire should be used to assist future assessment surveys of supermarket customer satisfaction.
\end{abstract}

Keywords: Supermarket; Questionnaire; Satisfaction; Lawshe.

\footnotetext{
${ }^{1}$ Universidade Candido Mendes - UCAM-Campos - Rua Anita Peçanha, 100, Parque São Caetano, Campos dos Goytacazes, RJ, CEP.: 28030-335, Brasil.

${ }^{2}$ Institutos Superiores de Ensino do CENSA - ISECENSA - Rua Salvador Correa, 139, Centro, Campos dos Goytacazes, RJ, CEP: 28035-310, Brasil.

${ }^{3}$ Instituto Federal Fluminense - IFF Centro - Rua Dr. Siqueira, 273, Parque Dom Bosco, Campos dos Goytacazes, RJ, CEP: 28030-130, Brasil.

(*) e-mail: franciscoleomachado@gmail.com

Data de recebimento: 08/11/2019. Aceito para publicação:17/02/2019.
}

Persp. Online: exatas \& eng., Campos dos Goytacazes, 27 (10) 43 - 53 - 2020

seer.perspectivasonline.com.br 


\section{INTRODUÇÃO}

A qualidade no atendimento é um fator determinante para manter uma empresa competitiva no mercado, dessa forma, o cliente passa a ser o foco da empresa. Silva et al. (2018) elaboram um plano de negócios buscando auxiliar empreendedores interssados em um mercado de microcervejaria artesanal, elaborando estratégias operacionais de marketing com a finalidade de explorar uma fatia do mercado de consumo de bebidas.

Kotler (2000, p.541) define supermercado como o "autosserviço que desenvolve operações relativamente grandes, de baixo custo, baixa margem e alto volume, projetado para atender a todas as necessidades de alimentação, higiene e limpeza doméstica".

Supermercado é o formato de varejo que possui maior destaque no sistema de autosserviço alimentar, é considerado atrativo tanto para os clientes, quanto os fornecedores de produtos. Por ser tratar de um local que atende as necessidades diárias dos clientes, possui visibilidade e alto número de visitas. Por apresentar grande variedade de itens, o setor supermercadista foi um dos poucos setores da economia brasileira que apresentaram um crescimento tão acelerado (BORGES, 2001).

O setor supermercadista brasileiro registrou faturamento de $\mathrm{R} \$ 355,7$ bilhões em 2018, um crescimento nominal de $0,7 \%$ em relação ao ano de 2017 , de acordo com a pesquisa Ranking ABRAS/SuperHiper. O resultado apresentado em 2018 representa 5,2\% do Produto Interno Bruto (PIB). A pesquisa destaca ainda que o setor encerrou o ano de 2018 com 89,6 mil lojas e 1,853 milhão de funcionários diretos, um aumento de 30,7 mil vagas de empregos no Brasil, em comparação ao ano de 2017 (ABRAS, 2019).

Nesse sentido, Picolo e Tontini (2008) afirmam que a satisfação dos clientes se tornou um dos principais fatores para o sucesso no mercado, devido à crescente competição entre as organizações. Para Salim e Sampaio (2006), a compreenção e a motivação do comportamento do consumidor auxilia nas decisões mercadológicas para elaborar estratégias de conquistar, manter e fidelizar os clientes.

Dessa forma, a empresa deve estar envolvida no atendimento do cliente com eficiência, pois, conforme Chiavenato (2014), elas existem para satisfazer as necessidades e expectativas dos clientes com a produção de bens ou serviços.

Este estudo é uma forma de proporcionar às empresas, principalmente do varejo supermercadista, uma visão sobre a importância da satisfação dos clientes. Neste sentido foi utilizado o método de Lawshe para validar os itens de um questionário sobre a satisfação de clientes em um supermercado na cidade de Campos dos Goytacazes - RJ.

\section{METODOLOGIA}

Foi utilizada a metodologia da pesquisa caracterizada como exploratória com levantamento bibliográfico baseados em materiais publicados na literatura, e como descritiva, descrevendo as características de uma determinada população ou estabelecendo relações entre as variáveis (GIL, 2018).

Persp. Online: exatas \& eng., Campos dos Goytacazes, 27 (10) 43 - 53 - 2020 


\subsection{Elaboração do Questionário}

A elaboração do questionário foi realizada por meio de embasamentos teóricos a fim de destacar, a partir de consultas em fontes de literaturas existentes (periódicos, teses, dissertações, congressos), os atributos considerados essenciais pelos clientes de um supermercado. Para definir os atributos do questionário foram selecionados vinte (20) trabalhos, dos quais foram identificados os itens mais frequentes, dos quais trinta (30) foram selecionados e agrupados em cinco dimensões: "Estrutura" ( 8 itens), "Atendimento" (9 itens), "Produtos" (7 itens), "Adicionais" (4 itens) e "Segurança" (2 itens) (Tabela 1).

Como instrumento de pesquisa e coleta de dados, utilizou-se o questionário estruturado com perguntas fechadas, com uma abordagem quantitativa, permitindo avaliar a importância dos atributos de satisfação de um supermercado em Campos dos Goytacazes, RJ, segundo a percepção dos clientes.

Inicialmente, foi realizado um pré-teste aplicando o questionário a dez clientes com o propósito de verificar a clareza e a adequação dos itens propostos. Segundo Malhotra (2006) o pré-teste tem a finalidade de avaliar o instrumento garantindo que meça o que se pretende, com intenção de identificar e eliminar os problemas em potencial. Posteriormente, o questionário foi avaliado por 48 especialistas (clientes) do supermercado em Campos dos Goytacaes-RJ, escolhidos de forma aleatória, e de acordo com a disponibilidade, para verificar se o instrumento contribuía para esclarecer o conceito da causa em estudo.

\subsection{Método de Lawshe}

O método de Lawshe (1975) tem como base a submissão de questionário estruturado, com perguntas fechadas, a especialistas (clientes), sendo que em cada item foi oferecido as seguintes opções de respostas: (1) não essencial; (2) essencial; e (NS) não sei/prefiro não opinar, segundo a percepção dos clientes de um supermercado em Campos dos Goytacazes-RJ. As respostas de cada item são agrupadas como "não essenciais" ou "essenciais" e foi verificada a validade de cada item, calculando uma taxa de validade de conteúdo, em inglês CVR (Content Validity Ratio), para cada um, conforme a seguinte fórmula:

$$
\mathrm{CRV}=\frac{\mathrm{ne}-\frac{\mathrm{N}}{2}}{\frac{\mathrm{N}}{2}}
$$

onde:"ne" é número de especialistas que classificaram cada item como "essencial" e "N" é o número total de respondentes, excluindo-se destes os que responderam "NS" não sei/prefiro não opinar".

A validação dos itens do questionário proposta por Lawshe (1975) foi modificada por Wilson e Pan e Schumsky (2012), que verificaram inconsistência nos cálculos propostos por Lawshe e publicou um artigo corrigindo a tabela original. Posteriormente, Ayre e Scally (2014) em seu estudo revisaram os métodos originais de cálculo do método de Lawshe, sugerindo métodos para cálculos iniciais de valores críticos e tabelas de probabilidades binomiais exatas. Logo para cada "N" foi então anotado o valor de CVRcrítico, adotando-se os valores revisados e calculados, utilizando-se o nível de significância de $5 \%$.

Persp. Online: exatas \& eng., Campos dos Goytacazes, 27 (10) 43 - 53 - 2020

seer.perspectivasonline.com.br 
Por fim, os valores de CVR calculados foram comparados com o valor mínimo do CVRcrítico. Para que o item fosse mantido no questionário o valor de CVRcalculado deveria superar o valor de CVRcrítico, proposto por Wilson, Pan e Schumsky (2014), caso contrário o item seria excluído do questionário. Esse procedimento também foi utilizado por Souza et al. (2015).

Tabela 1: Dimensões com seus, respectivos, itens de satisfação e seus autores correspondentes

\begin{tabular}{|c|c|c|c|c|c|c|c|c|c|c|}
\hline Dimensão / Item & & & & & $* \mathrm{Au}$ & tore: & & & & \\
\hline 1. Estrutura & A1 & A2 & A3 & A4 & A5 & A6 & A7 & A8 & A9 & $\mathbf{A 1 0}$ \\
\hline 1.1. Equipamentos em boas condições de uso & $\mathbf{x}$ & & & & & & & & & \\
\hline $\begin{array}{l}\text { 1.2. Equipamentos modernos (ex.: leitores de } \\
\text { código de barras) }\end{array}$ & $\mathbf{x}$ & & & & & & & & $\mathbf{x}$ & \\
\hline 1.3. Limpeza e higiene do ambiente & $\mathbf{x}$ & & & & $\mathbf{x}$ & & $\mathbf{x}$ & & $\mathbf{x}$ & \\
\hline 1.4. Iluminação e climatização adequada & $\mathbf{x}$ & & & & $\mathbf{x}$ & & $\mathbf{x}$ & $\mathbf{x}$ & & \\
\hline 1.5. Estrutura física com aparência agradável & & & & & & & & & & \\
\hline 1.6. Localização e fácil acesso & & & & $\mathbf{x}$ & & & $\mathbf{x}$ & $\mathbf{x}$ & $\mathbf{x}$ & \\
\hline 1.7. Estacionamento & $\mathbf{x}$ & & $\mathbf{x}$ & & $\mathbf{x}$ & $\mathbf{x}$ & $\mathbf{x}$ & $\mathbf{x}$ & & \\
\hline 1.8. Vagas preferenciais no estacionamento & & & & & & & & & & \\
\hline 2. Atendimento & & & & & & & & & & \\
\hline 2.1. Horário de funcionamento conveniente & & & & $\mathbf{x}$ & & & $\mathbf{x}$ & $\mathbf{x}$ & $\mathbf{x}$ & $\mathbf{x}$ \\
\hline $\begin{array}{l}\text { 2.2. Diversas formas de pagamento (cartões, ticket, } \\
\text { etc) }\end{array}$ & $\mathbf{x}$ & & & $\mathbf{x}$ & & & $\mathbf{x}$ & & & \\
\hline 2.3. Facilidades de pagamento (parcelamento) & & & & $\mathbf{x}$ & & & & & & \\
\hline 2.4. Agilidade no atendimento no caixa & $\mathbf{x}$ & & & $\mathbf{x}$ & & & & & $\mathbf{x}$ & \\
\hline $\begin{array}{l}\text { 2.5. Comunicação no atendimento de forma clara e } \\
\text { acessível }\end{array}$ & $\mathbf{x}$ & & & & & & & & & $\mathbf{x}$ \\
\hline $\begin{array}{l}\text { 2.6. Caixas exclusivos para idosos, gestantes e } \\
\text { deficientes. }\end{array}$ & $\mathbf{x}$ & & & & & & & & & \\
\hline 2.7. Qualidade no atendimento & $\mathbf{x}$ & & & $\mathbf{x}$ & & $\mathbf{x}$ & & & & $\mathbf{x}$ \\
\hline 2.8. Funcionários dispostos a ajudar e informar & $\mathbf{x}$ & & & & $\mathbf{x}$ & & & & & \\
\hline 2.9. Serviço de Atendimento ao Cliente (SAC) & $\mathbf{x}$ & $\mathbf{x}$ & & & & $\mathbf{x}$ & $\mathbf{x}$ & & $\mathbf{x}$ & $\mathbf{x}$ \\
\hline 3. Produtos & & & & & & & & & & \\
\hline 3.1. Organização dos produtos nas prateleiras & $\mathbf{x}$ & & $\mathbf{x}$ & & & $\mathbf{x}$ & & & $\mathbf{x}$ & \\
\hline 3.2. Grande variedade de produtos e marcas & $\mathbf{x}$ & & $\mathbf{x}$ & $\mathbf{x}$ & & $\mathbf{x}$ & $\mathbf{x}$ & $\mathbf{x}$ & $\mathbf{x}$ & $\mathbf{x}$ \\
\hline $\begin{array}{lllll}\begin{array}{l}\text { 3.3. Sinalização com a localização dos } \\
\text { departamentos }\end{array} & \text { a } \\
\end{array}$ & $\mathbf{x}$ & & & & & & & & & \\
\hline 3.4. Preços justos & & & & $\mathbf{x}$ & & & $\mathbf{x}$ & & $\mathbf{x}$ & \\
\hline 3.5. Etiquetas de preços nas mercadorias & $\mathbf{x}$ & & & & & & & & & \\
\hline 3.6. Produtos de qualidade & $\mathbf{x}$ & $\mathbf{x}$ & & $\mathbf{x}$ & & $\mathbf{x}$ & $\mathbf{x}$ & $\mathbf{x}$ & $\mathbf{x}$ & $\mathbf{x}$ \\
\hline 3.7. Disponibilidade e rápida reposição de produtos & $\mathbf{x}$ & & & & & & & & & \\
\hline 4. Adicionais & & & & & & & & & & \\
\hline 4.1. Ofertas e promoções atrativas & $\mathbf{x}$ & & $\mathbf{x}$ & $\mathbf{x}$ & $\mathbf{x}$ & $\mathbf{x}$ & $\mathbf{x}$ & & & $\mathbf{x}$ \\
\hline 4.2. Serviços no açougue e padaria & & & & & & & & $\mathbf{x}$ & & \\
\hline 4.3. Serviços de entrega em domicílio & $\mathbf{x}$ & & & & & $\mathbf{x}$ & & $\mathbf{x}$ & $\mathbf{x}$ & \\
\hline
\end{tabular}

4.4. Ações em datas comemorativas (sorteios e brindes)

\section{Segurança}

5.1. Serviços com garantia de segurança

5.2. Produtos com garantia de segurança

$\mathbf{x}$

$\mathbf{x}$

*Autores: A1 - Neubuser, Zamberlan e Sparemberger (2004); A2 - Melo (2015); A3 - Cesarino e Caixeta Filho (2002); A4 - Miranda (2001); A5 - Gallon et al. ((2008); A6 - Ribeiro, Kuki e Luz (2018); A7 - Silva (2017); A8 - Corrêa et al. (2017); A9 - Severgnini, Santos e Miura (2015); A10 - Margato (2014); 
Tabela 1: Dimensões com seus, respectivos, itens de satisfação e seus autores correspondentes (continuação)

\begin{tabular}{|c|c|c|c|c|c|c|c|c|c|c|}
\hline Dimensão / Item & & & & & *Aut & ores & & & & \\
\hline 1. Estrutura & A11 & A12 & A13 & A14 & A15 & A16 & A17 & A18 & A19 & A20 \\
\hline 1.1. Equipamentos em boas condições de uso & & & & $\mathbf{x}$ & & & & & & \\
\hline $\begin{array}{l}\text { 1.2. Equipamentos modernos (ex.: leitores de } \\
\text { código de barras) }\end{array}$ & & & & $\mathbf{x}$ & & & & $\mathbf{x}$ & & \\
\hline 1.3. Limpeza e higiene do ambiente & & & $\mathbf{x}$ & $\mathbf{x}$ & & $\mathbf{x}$ & & $\mathbf{x}$ & $\mathbf{x}$ & \\
\hline 1.4. Iluminação e climatização adequada & & & & & $\mathbf{x}$ & & & $\mathbf{x}$ & & \\
\hline 1.5. Estrutura física com aparência agradável & & & & $\mathbf{x}$ & $\mathbf{x}$ & & & $\mathbf{x}$ & $\mathbf{x}$ & \\
\hline 1.6. Localização e fácil acesso & & & & $\mathbf{x}$ & & & $\mathbf{x}$ & $\mathbf{x}$ & $\mathbf{x}$ & \\
\hline 1.7. Estacionamento & & & $\mathbf{x}$ & $\mathbf{x}$ & & & $\mathbf{x}$ & & & \\
\hline 1.8. Vagas preferenciais no estacionamento & $\mathbf{x}$ & & & & & & & & & \\
\hline 2. Atendimento & & & & & & & & & & \\
\hline 2.1. Horário de funcionamento conveniente & & & & $\mathbf{x}$ & & & $\mathbf{x}$ & & & \\
\hline $\begin{array}{l}\text { 2.2. Diversas formas de pagamento (cartões, } \\
\text { ticket, etc) }\end{array}$ & & & & $\mathbf{x}$ & & & & & $\mathbf{x}$ & \\
\hline 2.3. Facilidades de pagamento (parcelamento) & & & & $\mathbf{x}$ & & & $\mathbf{x}$ & & & \\
\hline 2.4. Agilidade no atendimento no caixa & & & & & & & & $\mathbf{x}$ & $\mathbf{x}$ & \\
\hline $\begin{array}{l}\text { 2.5. Comunicação no atendimento de forma } \\
\text { clara e acessível }\end{array}$ & & & & $\mathbf{x}$ & $\mathbf{x}$ & & & $\mathbf{x}$ & $\mathbf{x}$ & \\
\hline $\begin{array}{l}\text { 2.6. Caixas exclusivos para idosos, gestantes e } \\
\text { deficientes. }\end{array}$ & & & & & & & & $\mathbf{x}$ & & \\
\hline 2.7. Qualidade no atendimento & & & $\mathbf{x}$ & $\mathbf{x}$ & $\mathbf{x}$ & $\mathbf{x}$ & & $\mathbf{x}$ & $\mathbf{x}$ & \\
\hline 2.8. Funcionários dispostos a ajudar e informar & & & & $\mathbf{x}$ & $\mathbf{x}$ & & & $\mathbf{x}$ & $\mathbf{x}$ & \\
\hline 2.9. Serviço de Atendimento ao Cliente (SAC) & & & & & & & & $\mathbf{x}$ & & \\
\hline 3. Produtos & & & & & & & & & & \\
\hline 3.1. Organização dos produtos nas prateleiras & & & & & & $\mathbf{x}$ & & $\mathbf{x}$ & & $\mathbf{x}$ \\
\hline 3.2. Grande variedade de produtos e marcas & & $\mathbf{x}$ & $\mathbf{x}$ & & & & & $\mathbf{x}$ & $\mathbf{x}$ & $\mathbf{x}$ \\
\hline $\begin{array}{l}\text { 3.3. Sinalização com a localização dos } \\
\text { departamentos }\end{array}$ & & & $\mathbf{x}$ & $\mathbf{x}$ & & & & & $\mathbf{x}$ & \\
\hline 3.4. Preços justos & & $\mathbf{x}$ & & & $\mathbf{x}$ & $\mathbf{x}$ & & $\mathbf{x}$ & $\mathbf{x}$ & $\mathbf{x}$ \\
\hline 3.5. Etiquetas de preços nas mercadorias & & & & & & & & & $\mathbf{x}$ & \\
\hline 3.6. Produtos de qualidade & & $\mathbf{x}$ & & & $\mathbf{x}$ & $\mathbf{x}$ & $\mathbf{x}$ & & $\mathbf{x}$ & $\mathbf{x}$ \\
\hline $\begin{array}{l}\text { 3.7. Disponibilidade e rápida reposição de } \\
\text { produtos }\end{array}$ & & & & $\mathbf{x}$ & & & & $\mathbf{x}$ & $\mathbf{x}$ & \\
\hline 4. Adicionais & & & & & & & & & & \\
\hline 4.1. Ofertas e promoções atrativas & & & & $\mathbf{x}$ & & $\mathbf{x}$ & $\mathbf{x}$ & & $\mathbf{x}$ & $\mathbf{x}$ \\
\hline 4.2. Serviços no açougue e padaria & & & & $\mathbf{x}$ & & & & $\mathbf{x}$ & & \\
\hline 4.3. Serviços de entrega em domicílio & & & $\mathbf{x}$ & & & & & & $\mathbf{x}$ & \\
\hline $\begin{array}{l}\text { 4.4. Ações em datas comemorativas (sorteios e } \\
\text { brindes) }\end{array}$ & & & & $\mathbf{x}$ & & & & & & \\
\hline 5. Segurança & & & & & & & & & & \\
\hline 5.1. Serviços com garantia de segurança & & & $\mathbf{x}$ & $\mathbf{x}$ & & & & & & \\
\hline 5.2. Produtos com garantia de segurança & & & $\mathbf{x}$ & $\mathbf{x}$ & & & & & & \\
\hline
\end{tabular}

* Autores: A11 - Silva (2013); A12 - Oliveira (2013); A13 - Gallon et al. (2007); A14 - Borges (2001); A15 Kotler (2000); A16 - Silva (2012); A17 - Queiroz (2008); A18 - Prado e Marchetti (1997); A19 - Blanco e Moraga (2003); A20 - Pereira (2001). 


\section{RESULTADOS E DISCUSSÃO}

Para validação dos itens do questionário sobre a satisfação dos clientes em um supermercado, os resultados foram discutidos por cada dimensão.

Na tabela 2 encontram-se os oito itens da dimensão "Estrutura", avaliados pelos 48 especialistas (clientes).

Tabela 2: Dimensão "Estrutura" e itens correspondentes

\begin{tabular}{l|c|c|c|c|c|c}
\hline Dimensão/Item & ne & $\mathrm{N}$ & $\%$ essenciais & $\mathrm{CVR}_{\text {calc }}$ & $\mathrm{CVR}_{\text {crít }}$ & Decisão \\
\hline $\begin{array}{l}\text { 1. Estrutura } \\
\text { 1.1. Equipamentos em boas condições de uso }\end{array}$ & 47 & 47 & $100,0 \%$ & 1,000 & 0,286 & Manter \\
\hline $\begin{array}{l}\text { 1.2. Equipamentos modernos (ex.: leitores de } \\
\text { código de barras) }\end{array}$ & 46 & 47 & $97,9 \%$ & 0,957 & 0,286 & Manter \\
\hline 1.3. Limpeza e higiene do ambiente & 48 & 48 & $100,0 \%$ & 1,000 & 0,283 & Manter \\
\hline 1.4. Iluminação e climatização adequada & 47 & 48 & $97,9 \%$ & 0,958 & 0,283 & Manter \\
\hline 1.5. Estrutura física com aparência agradável & 42 & 45 & $93,3 \%$ & 0,867 & 0,292 & Manter \\
\hline 1.6. Localização e fácil acesso & 43 & 47 & $91,5 \%$ & 0,830 & 0,286 & Manter \\
\hline 1.7. Estacionamento & 40 & 48 & $83,3 \%$ & 0,667 & 0,283 & Manter \\
\hline 1.8. Vagas preferenciais no estacionamento & 41 & 47 & $87,2 \%$ & 0,745 & 0,286 & Manter \\
\hline
\end{tabular}

ne: número de participantes que avaliaram o item como essencial; $\mathrm{N}$ : número total de partcipantes excluindo aqueles que responderam NS: não sei/prefiro não opinar; $\mathrm{CVR}_{\text {calc }}$ : CVRcalculado; $\mathrm{CVR}_{\text {crit }}$ : CVRcrítico; Decisão: manter ou excluir os itens.

Na dimensão "1. Estrutura", todos os dados foram mantidos, visto que para todos o valor do CVRcalculado é maior que o CVRcrítico. Dos atributos relacionados à estrutura física do supermercado, os itens "1.1. Equipamentos em boas condições de uso" e "1.3. Limpeza e higiene do ambiente" foram considerados $100 \%$ essenciais, segundo a percepção dos clientes, corroborando com os autores Neubuser, Zamberlan e Sparemberger (2004) e Borges (2001). O item "1.6. Localização e fácil acesso" e o item "1.7. Estacionamento" concordam com as pesquisas dos autores Silva (2017), Corrêa et al. (2017) e Queiroz (2008). Além desses, o item "1.2. Equipamentos modernos (ex.: leitores de código de barras)" apresentou concordância com o trabalho de Severgnini, Santos e Miura (2015) e Prado e Marchetti (1997).

Pode-se observar na tabela 3 os nove itens da dimensão "Atendimento", avaliados pelos 48 especialistas (clientes).

Nesta dimensão, todos os itens devem ser mantidos sendo que os itens "2.2. Diversas formas de pagamento (cartões, ticket, etc)" e "2.9. Serviço de Atendimento ao cliente (SAC)", corroboraram com os autores Neubuser; Zamberlan; Sparemberger (2004) e Silva (2017); e o item "2.5. Comunicação no atendimento de forma clara e acessível" que está de acordo com os autores Margato (2014) e Kotler (2000), pois apresentam o valor do CVRcalculado superior ao valor do CVRcrítico. 
Tabela 3: Dimensão "Atendimento" e itens correspondentes

\begin{tabular}{l|c|c|c|c|c|c}
\hline Dimensão/Item & $\mathrm{ne}$ & $\mathrm{N}$ & $\%$ essenciais & CVR $_{\text {calc }}$ & CVR $_{\text {crít }}$ & Decisão \\
\hline 2. Atendimento & & & & & & \\
\hline 2.1. Horário de funcionamento conveniente & 47 & 48 & $97,9 \%$ & 0,958 & 0,283 & Manter \\
\hline $\begin{array}{l}\text { 2.2. Diversas formas de pagamento (cartões, } \\
\text { ticket, etc) }\end{array}$ & 48 & 48 & $100,0 \%$ & 1,000 & 0,283 & Manter \\
\hline 2.3. Facilidades de pagamento (parcelamento) & 35 & 47 & $74,5 \%$ & 0,489 & 0,286 & Manter \\
\hline 2.4. Agilidade no atendimento no caixa & 48 & 48 & $100,0 \%$ & 1,000 & 0,283 & Manter \\
\hline $\begin{array}{l}\text { 2.5. Comunicação no atendimento de forma } \\
\text { clara e acessível }\end{array}$ & 48 & 48 & $100,0 \%$ & 1,000 & 0,283 & Manter \\
\hline $\begin{array}{l}\text { 2.6. Caixas exclusivos para idosos, gestantes e } \\
\text { deficientes }\end{array}$ & 45 & 48 & $93,8 \%$ & 0,875 & 0,283 & Manter \\
\hline 2.7. Qualidade no atendimento & 48 & 48 & $100,0 \%$ & 1,000 & 0,283 & Manter \\
\hline 2.8. Funcionários dispostos a ajudar e informar & 48 & 48 & $100,0 \%$ & 1,000 & 0,283 & Manter \\
\hline 2.9. Serviço de Atendimento ao Cliente (SAC) & 41 & 48 & $85,4 \%$ & 0,708 & 0,283 & Manter \\
\hline
\end{tabular}
ne: número de participantes que avaliaram o item como essencial; N: número total de partcipantes excluindo aqueles que responderam NS: não sei/prefiro não opinar; $\mathrm{CVR}_{\text {calc: }}$ CVRcalculado; $\mathrm{CVR}_{\text {crit: }}$ CVRcrítico; Decisão: manter ou excluir os itens.

$\mathrm{Na}$ tabela 4 estão os sete itens da dimensão "Produto", avaliados pelos 48 especialistas (clientes).

Tabela 4: Dimensão "Produto" e itens correspondentes

\begin{tabular}{|c|c|c|c|c|c|c|}
\hline Dimensão/Item & ne & $\mathrm{N}$ & \%essenciais & $\mathrm{CVR}_{\mathrm{calc}}$ & $\mathrm{CVR}_{\text {crít }}$ & Decisão \\
\hline \multicolumn{7}{|l|}{ 3. Produtos } \\
\hline 3.1. Organização dos produtos nas prateleiras & 48 & 48 & $100,0 \%$ & 1,000 & 0,283 & Manter \\
\hline 3.2. Grande variedade de produtos e marcas & 45 & 47 & $95,7 \%$ & 0,915 & 0,286 & Manter \\
\hline $\begin{array}{l}\text { 3.3. Sinalização com a localização dos } \\
\text { departamentos }\end{array}$ & 42 & 46 & $91,3 \%$ & 0,826 & 0,289 & Manter \\
\hline 3.4. Preços justos & 48 & 48 & $100,0 \%$ & 1,000 & 0,283 & Manter \\
\hline 3.5. Etiquetas de preços nas mercadorias & 47 & 48 & $97,9 \%$ & 0,958 & 0,283 & Manter \\
\hline 3.6. Produtos de qualidade & 48 & 48 & $100,0 \%$ & 1,000 & 0,283 & Manter \\
\hline 3.7. Disponibilidade e rápida reposição de produtos & 47 & 48 & $97,9 \%$ & 0,958 & 0,283 & Manter \\
\hline
\end{tabular}

$\mathrm{Na}$ dimensão "4. Produtos", todos os itens presentes no questionário (Tabela 4) foram mantidos levando em consideração que os CVRcalculados foram maiores que os CVRcríticos. Avaliando a condição dos "Produtos" e a forma de apresentá-los no supermercado, destacam-se os itens "3.1. Organização dos produtos nas prateleiras", "3.4. Preços justos" e "3.6. Produtos de qualidade", pois foram unânimes na pesquisa, corroborando com os autores Oliveira (2013) e Pereira (2001).

A tabela 5 refere-se aos quatro itens da dimensão "Adicionais", avaliados pelos 48 especialistas (clientes).s

Já a dimensão "5. Adicionais" exibe itens que serão mantidos por constar o CVRcalculado maior que o CVRcrítico, como "4.1 Ofertas e promoções atrativas" e "4.2 Serviços no açougue e padaria" concordando com Borges (2001). Porém, nessa mesma dimensão ressaltam-se dois itens nos quais suas essencialidades não foram confirmadas, o item "4.3. Serviços de entrega em domicílio" não corroborando com os resultados de

Persp. Online: exatas \& eng., Campos dos Goytacazes, 27 (10) 43 - 53 - 2020

seer.perspectivasonline.com.br 
Ribeiro, Kuki e Luz (2018), Gallon et al. (2007) e Blanco e Moraga (2003) e o item "4.4. Ações em datas comemorativas (sorteios e brindes)" que foi pouco citado nos trabalhos pesquisados e não corrobora com Borges (2001), sendo assim, ambos devem ser excluídos do questionário.

Tabela 5: Dimensão “Adicionais" e itens correspondentes

\begin{tabular}{lcccccc}
\hline Dimensão/Item & ne & $\mathrm{N}$ & $\%$ essenciais & CVR $_{\text {calc }}$ & CVR $_{\text {crít }}$ & Decisão \\
\hline 4. Adicionais & & & & & & \\
\hline 4.1. Ofertas e promoções atrativas & 45 & 48 & $93,8 \%$ & 0,875 & 0,283 & Manter \\
\hline 4.2. Serviços no açougue e padaria & 44 & 48 & $91,7 \%$ & 0,833 & 0,283 & Manter \\
\hline 4.3. Serviços de entrega em domicílio & 28 & 46 & $60,9 \%$ & 0,217 & 0,289 & Excluir \\
\hline 4.4. Ações em datas comemorativas (sorteios e brindes) & 25 & 48 & $52,1 \%$ & 0,042 & 0,283 & Excluir \\
\hline ne: número de participantes que avaliaram o item como essencial; N: número total de partcipantes excluindo \\
$\begin{array}{l}\text { aqueles que responderam NS: não sei/prefiro não opinar; CVR calc: CVRcalculado; CVR } \\
\text { Decitisão: CVRcrítico; }\end{array}$
\end{tabular}

Observa-se na tabela 6 os dois itens da dimensão "Segurança", avaliados pelos 48 especialistas (clientes).

Tabela 6: Dimensão "Segurança" e itens correspondentes

\begin{tabular}{|c|c|c|c|c|c|c|}
\hline Dimensão/Item & ne & $\mathrm{N}$ & \%essenciais & $\mathrm{CVR}_{\text {calc }}$ & CVR $_{\text {crít }}$ & Decisão \\
\hline \multicolumn{7}{|l|}{ 5. Segurança } \\
\hline 5.1. Serviços com garantia de segurança & 48 & 48 & $100,0 \%$ & 1,000 & 0,283 & Manter \\
\hline 5.2. Produtos com garantia de segurança & 48 & 48 & $100,0 \%$ & 1,000 & 0,283 & Manter \\
\hline
\end{tabular}

Fonte: Própria.

Por fim, na dimensão "5. Segurança", os itens "5.1. Serviços com garantia de segurança" e "5.2. Produtos com garantia de segurança" foram considerados totalmente essenciais, segundo a percepção dos clientes, concordando com os autores Neubuser; Zamberlan; Sparemberger (2004), Gallon et al. (2007) e Borges (2001).

\section{CONCLUSÕES}

Foi possível verificar a validade do questionário composto por 30 itens e distribuídos em cinco dimensões por meio da aplicação do método de Lawshe. Atributos como "Limpeza e higiene do ambiente", "Diversas formas de pagamentos (cartões, ticket, etc)", "Agilidade no atendimento no caixa", "Qualidade no atendimento", "Funcionários dispostos a ajudar e informar", "Preços justos", "Produtos de qualidade", "Organização dos produtos nas prateleiras", "Produtos com garantia de segurança" são alguns dos atributos mais valorizados pelos consumidores, obtendo $100 \%$ de essencialidade. Por outro lado, os atributos "Serviço de entrega em domicílio" e "Ações em datas comemorativas (sorteios e brindes)", não foram considerados essenciais, segundo a percepção dos clientes participantes da pesquisa, indicando que os itens mantidos no questionário, com a aplicação do método de Lawshe, podem ser utilizados para auxiliar futuras pesquisas de avaliação da satisfação de clientes de supermercados em um município, uma vez que a qualidade e a satisfação dos clientes são fatores determinantes para o sucesso de uma empresa.

Persp. Online: exatas \& eng., Campos dos Goytacazes, 27 (10) 43 - 53 - 2020

seer.perspectivasonline.com.br 


\section{REFERÊECIAS}

ABRAS. SETOR SUPERMERCADISTA FATURA R\$ 355,7 BILHÕES EM 2018. 2019. Elaborada por Redação Portal ABRAS. Disponível em: $<$ http://www.abras.com.br/clipping.php?area=20\&clipping=67505>. Acesso em: 25 abr. 2019.

AYRE, C.; SCALLY, A. J. Critical values for Lawshe's content validity ratio: revisiting the original methods of calculation. Measurement and Evaluation in Counseling and Development, v. 47, n. 1, p. 79-86, 2014. DOI: 10.1177/0748175613513808

BLANCO, C. F.; MORAGA, E. T.. Factores relacionados con la lealtad: Un análisis del compromiso con la compra y de los atributos de los supermercados. Theoria, v. 12, n. 1, p. 97-106, 2003.

BORGES, A. R. Marketing de varejo: As estratégias adotadas pelos supermercados de vizinhança para conquistar e fidelizar clientes. 2001. 173 f. Monografia (Especialização) Curso de Engenharia de Produção, Universidade Federal de Santa Catarina, Florianópolis, 2001.

CESARINO, R. C.; CAIXETA FILHO, J. V. Alocação dos produtos nas gôndolas dos supermercados: um estudo de caso. Gestão \& Produção, São Carlos, v. 9, n. 1, p.45-61, abr. 2002. FapUNIFESP (SciELO). DOI: 10.1590/s0104-530x2002000100005

CHIAVENATO, I. Administração nos novos tempos: os novos horizontes em administração. 3. ed. Barueri, SP: Manole, 2014.

CORRÊA, L. A. et al. Nível de satisfação de clientes: estudo de casos em um supermercado em Itaocara, RJ. In: SIMPÓSIO DE ENGENHARIA DE PRODUÇÃO, 1., 2017, Catalão. Anais... . Catalão: Universidade Federal de Goiás, 2017. p. 1 - 9.

GALLON, A. V., DA SILVA, T. P., HEIN, N., \& OLINQUEVITCH, J. L. Avaliação da importância dos atributos ea satisfação dos clientes de supermercados do Médi Vale do Itajaí-SC. RBGN: Revista Brasileira de Gestão de Negócios, v. 10, n. 27, p. 150-160, 2008.

GIL, A. C. Como elaborar projetos de pesquisa. 6. ed. São Paulo: Atlas, 2018.

KOTLER, P. Administração de Marketing. São Paulo: Ed. Prentice Hall, 2000.

LAWSHE, C. H. A quantitative approach to content validity 1. Personnel psychology, v. 28, n. 4, p. 563-575, 1975. DOI: 10.1111/j.1744-6570.1975.tb01393.x

MAlHOTRA, N. K. Pesquisa em marcheting: uma orientação aplicada. 4. ed. Porto Alegre: Ed. Bookman, 2006.

MARGATO, K. C. A. A qualidade de serviço: um estudo sobre a satisfação do cliente do Intermarché de Cantanhede. 2014. 91 f. Dissertação (Mestrado) - Curso de Administração Pública Empresarial, Faculdade de Direito da Universidade de Coimbra, Coimbra, 2014.

MELO, D.C.; ALCÂNTARA, R. L. C. Um modelo da maturidade da gestão da demanda: Um estudo multicaso na cadeia de suprimento de produtos de mercearia básica. Gestão \&

Persp. Online: exatas \& eng., Campos dos Goytacazes, 27 (10) 43 - 53 - 2020

seer.perspectivasonline.com.br 
Produção, São Carlos, v. 22, n. 1, p.53-66, mar. 2015. FapUNIFESP (SciELO). DOI: 10.1590/0104-530x0701-13.

MIRANDA, C. L. Satisfação do cliente em supermercados: a avaliação da qualidade dos serviços. 2001. 182 f. Dissertação (Mestrado) - Curso de Engenharia de Produção, Universidade Federal de Santa Catarina, Florianópolis, 2001.

NEUBUSER, M. E.; ZAMBERLAN, L.; SPAREMBERGER, A. A satisfação do consumidor de supermercados. Revista de Administração, v. 3, n. 4, p. 41-65, 2004.

OLIVEIRA, L. D. S.; LIMA-FILHO, D. O.; WATANABE, E. A. M. Nível de desenvolvimento e tecnologia de distribuição de alimentos em países selecionados. Revista de Economia e Sociologia Rural, v. 51, n. 1, p. 09-24, 2013. DOI: 10.1590/S0103-20032013000100001

PEÇANHA, J.; SHIMODA, E.; SHIMOYA, A.; \& RADAEL, M. C. Avaliação de pizzarias sob a percepção dos clientes: determinação da validade de itens do questionário. Humanas \& Sociais Aplicadas, v. 5, n. 13, 2015.

PEREIRA, I. Marcas de supermercado. Revista de Administração de Empresas, São Paulo, v. 41, n. 1, p.16-27, mar. 2001.

PICOLO, J. D.; TONTINI, G. Análise do contraste da penalidade e da recompensa (PRC): identificando oportunidades de melhoria em um serviço. RAM. Revista de Administração Mackenzie, v. 9, n. 5, p. 35-58, 2008. DOI: 10.1590/S1678-69712008000500003

PRADO, P. H. M.; MARCHETTI, R. Z. Excelência em supermercados: a dimensão da satisfação do consumidor. Revista de Administração, v. 32, n. 2, p. 58-64, 1997.

QUEIROZ, T. S.; SOUZA, T.; GOUVINHAS, R. P. Estratégias de marketing como um diferencial na escolha do supermercado de bairro. RAM. Revista de Administração Mackenzie, São Paulo, v. 9, n. 3, p.58-84, jun. 2008. DOI: 10.1590/S167869712008000300004

RIBEIRO, F. A.; KUKI, A. K.; LUZ, B. E. Adamowicz da. Análise da Satisfação de Clientes em um Supermercado. In: CONGRESSO BRASILEIRO DE ENGENHARIA DE PRODUÇÃO, 8., 2018, Ponta Grossa. Anais. Ponta Grossa: Aprepo, 2018. p. 1 - 12.

SALIM, C. J.; SAMPAIO, D. O. Fatores que influenciam os consumidores de Juiz de Fora a optarem por um determinado supermercado. Juiz de Fora: Estação Científica, n. 3, 2006.

SEVERGNINI, F.; SANTOS, V.; ABREU, J. C. A SATISFAÇÃO DO CLIENTE: UM ESTUDO NO MERCADO ALFA. Revista eletrônica Ciências da Administração e Turismo, v. 3, n. 3, 2016.

SILVA, I. G. B.; MINE, T.; REI, M.; OLIVEIRA, A. L.; DE PAULA, E.; \& CHAVES, S. Importância dos atributos de escolha entre varejos supermercadistas: uma pesquisa quantitativa com consumidores de Jacareí-SP.Revista de Administração e Negócios da Amazônia, v. 9, n. 3, p. 163-178, 2017. DOI: 10.18361/2176-8366/rara.v9n3p163-178

Persp. Online: exatas \& eng., Campos dos Goytacazes, 27 (10) 43 - 53 - 2020 seer.perspectivasonline.com.br 
SILVA, L. A.; MERLO, E. M.; NAGANO, M. S. Uma análise dos principais elementos influenciadores da tomada de decisão de compra de produtos de marca própria de supermercados. REAd. Revista Eletrônica de Administração (Porto Alegre), v. 18, n. 1, p. 97-129, 2012. DOI: 10.1590/S1413-23112012000100004

SILVA, R. F. C.; PINHEIRO, E. M.; SERRA, M. C.; RODRIGUES, A. D. L. P.; \& VIEIRA, A. C. S. Plano de Negócios de uma Microcervejaria Artesanal, Maranhão. Exatas \& Engenharias, v. 8, n. 21, 2018. DOI: 10.25242/885X82120181133

SILVA, T. I. Y. Desenvolvimento humano social e lazer: um estudo sobre a vivência do lazer pela terceira idade na cidade de Franca/SP. 2013. 144 f. Dissertação (Mestrado) Curso de Desenvolvimento Regional, Centro Universitário de Franca - Uni - Facef, Franca, 2013.

WILSON, F. R.; PAN, W.; SCHUMSKY, D. A. Recalculation of the critical values for Lawshe's content validity ratio. Measurement and evaluation in counseling and development, v. 45, n. 3, p. 197-210, 2012. DOI: 10.1177/0748175612440286 